\title{
Tripartite siRNA Micelles as Controlled Delivery Systems for Primary Dendritic Cells
}

\author{
Ariane Boudier, Anne Aubert-Pouëssel, Corine Gérardin, Jean-Marie Devoisselle, \\ and Sylvie Bégu \\ Institut Charles Gerhardt Montpellier, UMR 5253 CNRS-ENSCM-UM2-UM1, Montpellier, France
}

\author{
Pascale Louis-Plence and Julie Quentin \\ Inserm, U 844, MONTPELLIER, INM Hôpital, Montpellier, France; Université MONTPELLIER1, \\ Montpellier, France
}

Christian Jorgensen

Inserm, U 844, MONTPELLIER, INM Hôpital, Montpellier, France; Université MONTPELLIER1, Montpellier, France; CHU Hôpital Lapeyronie, Unité Clinique d'Immuno-rhumatologie: Thérapeutique des maladies articulaires et osseuses, Montpellier, France

\begin{abstract}
Dendritic cells (DCs) are key cells in immunology that are able to stimulate or inhibit the immune response. RNA interference has appeared of great interest to modulate the expression of immunogenic or tolerogenic molecules. In our study, pH-sensitive polyion complex micelles based on a double-hydrophilic block copolymer and poly-L-lysine were formulated to entrap a small interfering RNA (siRNA). We show that siRNA-loaded micelles were cytotolerant and efficiently endocytosed by DCs. siRNA targeting eGFP, used as model siRNA, was released into the cytosol following endocytosis of the micelles and the silencing of eGFP expression was observed in DC isolated from transgenic mice.

Our results underscore the potential of $\mathrm{pH}$-sensitive polyion complex micelles to formulate therapeutic siRNA for DC engineering in order to maintain the homeostasis of the immune response.
\end{abstract}

Keywords siRNA; pH-sensitive polyion complex micelles; tripartite association; dendritic cells; endosomal escape; gene silencing

\section{INTRODUCTION}

The first work describing RNA interference was published a few years ago on plant cells (Napoli, Lemieux, \& Jorgensen, 1990) and then on eukaryotic cells (Fire et al., 1998). This natural mechanism of RNA interference occurs in gene expression regulation thanks to a small interfering RNA (siRNA)

Address correspondence to Sylvie Bégu, Institut Charles Gerhardt Montpellier, UMR 5253 CNRS-ENSCM-UM2-UM1, 8 rue de l'Ecole Normale, F-34296 Montpellier, France. E-mail: sylvie.begu@enscm.fr composed of double strand consisting of 19-21 nucleotides with two or four nucleotide overhangs at the $3^{\prime}$ ends ( $\mathrm{Li}$ et al., 2006). siRNA interaction with the multi-protein complex RNA-induced silencing complex (RISC) leads to RNA dehybridation and to the cleavage of the targeted mRNA. This discovery has offered much hope since RNA interference is highly specific and versatile. Indeed, siRNA can theoretically be directed toward any gene and be efficient with, in many cases, over $90 \%$ of silencing. Nevertheless, the use of siRNA as therapeutic agent is a major challenge because of its poor stability, with a half-life of less than an hour (Li et al., 2006), explained by nuclease degradation (Akhtar \& Benter, 2007). In this way, various vectors have been tested for years: viral vectors, such as retrovirus, lentivirus, adeno-associated virus, and baculovirus (Li et al., 2006); and synthetic vectors, such as cationic liposomes, polymeric nanoparticles (Zhang, Zhao, Jiang, Wang, \& Ma, 2007), and micelles (Nishiyama \& Kataoka, 2006). Viral vectors usually offer high tranfection efficiencies on many cell lines, nonetheless, main problems concerning safety with potential mutation and oncogenic effect remain (Zhang et al., 2007). As far as the synthetic vectors are concerned, block copolymer micelles have already been used for RNA interference (Nishiyama \& Kataoka, 2006; Oishi et al., 2005). These core-shell objects usually present a narrow size distribution centered on a few tens of nanometers and are characterized by a stealth behavior, an ability to accumulate into inflammatory or cancerous tissues (Lavasanifar, Samuel, \& Kwon, 2002), and finally a renal elimination because of their molecular weight lower than the threshold of glomerular filtration (Kakizawa \& Kataoka, 2002). Properties of sensitivity to 
environmental parameters, such as $\mathrm{pH}$ or ionic strength, can be given to micelles if they are formulated with specific doublehydrophilic block copolymers. Those properties give micelles the possibility to release drugs into specific tissues or organelles (Nishiyama \& Kataoka, 2006). In a previous work, $\mathrm{pH}$-sensitive block copolymer micelles were formulated showing an uncommon biocompatibility toward dendritic cells (DCs) (Boudier et al., 2009). This result allows us to envisage cell therapy using such engineered cells. This is of great interest as DCs may stimulate the immune response in cancer or modulate the autoimmune response as a function of their maturation state (Hart, 1997; Steinman \& Banchereau, 2007). Nonetheless, up to now, only few works describing interference applied to DCs have been exposed in the literature (Hill et al., 2003; Li et al., 2007). In this study, we aimed at exploiting micelles, resulting from a tripartite association between a block copolymer with a polymethacrylic acid block, a counter polyion, poly-L-lysine (PLL) and a model siRNA, as tools that are able to deliver nucleotides into cell cytoplasm in order to induce gene expression silencing.

\section{MATERIALS AND METHODS}

\section{Materials}

siRNA-TAMRA sequences (sense, GGCUACGUCCAGGAGCGCAtt; antisense, UGCGCUCCUGGACGUAGCCtt) were synthesized and annealed according to the manufacturer specifications (Curevac, Tubingen, Germany). The sense strand was labelled with carboxytetramethylrhodamine (TAMRA) on $5^{\prime}$ end. siRNA sequences targeting eGFP were sense 5'HGCAAGCUGACCCUGAAGUUCATT and antisense 5'HUGAACUUCAGGGUCAGCUUGCTT for the control. PMAA ${ }_{2100}$-b-POE 5000 (P4386A-EOMAA) was obtained from Polymer Source (Dorval, Canada). PLL having molecular weight between 15,000 and $30,000 \mathrm{~g} / \mathrm{mol}$ and fluorescein isothyocyanate (FITC)-linked PLL (PLL-FITC) for micelle formation and other reagents used in the study were obtained from Sigma Aldrich (Saint Quentin Fallavier, France). Water was purified with a Milli-Q purification system (Millipore, Saint Quentin en Yvelines, France).

\section{Micelle Preparation}

For the blank micelle synthesis, two solutions were prepared in phosphate buffer saline (PBS) solution $(150 \mathrm{mM}, \mathrm{pH}=7.4)$ : polymethacrylic acid- $b$-polyethylene oxide (PMAA- $b$-POE) copolymer at $1.3 \mathrm{mg} / \mathrm{mL}$ and PLL at $0.73 \mathrm{mg} / \mathrm{mL}$. The mixture was realized by adding the same volume of the two components so as to be at a ratio of charges, $R=\left(\mathrm{NH}_{2}\right) /(\mathrm{COOH})$, of 1 . The final suspension was then shaken at room temperature (RT) for $3 \mathrm{~h}$.

Two kinds of siRNA-loaded micelles were prepared depending of the nature of the siRNA: siRNA-TAMRA or siRNA-eGFP using the protocol described above (ratio 1). Three protocols were tested for the encapsulation of siRNA used from 0.1 to $5 \mathrm{nmol} / \mathrm{mL}$ : (i) siRNA was first diluted in PLL solution before adding the copolymer solution; (ii) siRNA was first diluted in the copolymer solution before adding the PLL solution; and (iii) siRNA was added into the micelles suspension after the 3-h incubation. Finally, a filtration/centrifugation step was performed in order to separate nonencapsulated siRNA from siRNA-loaded micelles using VectaSpin Micro filters (12,000 MWCO) (Whatman, Versailles, France) and a centrifuge Sigma 2K15 (Fisher Bioblock, France).

\section{Micelle Characterization \\ Dynamic Light Scattering}

Dynamic light scattering (DLS) was used to determine scattered intensities, hydrodynamic diameters $(\mathrm{Dh})$, and polydispersity index using a Malvern Autosizer 4800 (Malvern, UK). The CONTIN program was used to extract size distributions from the autocorrelation functions. The volume-averaged values of the Dh were given in this study.

\section{UV Spectrophotometer}

siRNA encapsulation yield was performed thanks to a standard quantification by absorbance at $260 \mathrm{~nm}$ using a Lambda 35 spectrophotometer (Perkin Elmer, Courtaboeuf, France).

\section{Pyrene Fluorescence Study}

Pyrene, a highly hydrophobic molecule, serves as a probe to highlight hydrophobic domains. In this way, pyrene incorporation was studied in copolymer aqueous solutions at various $\mathrm{pH}$ with a final concentration of $2 \times 10^{-6} \mathrm{M}$. Fluorescence spectra were recorded using excitation wavelength at $340 \mathrm{~nm}$ and emission wavelength at 373 and $384 \mathrm{~nm}$ (corresponding, respectively, to first and third vibronic bands I1 and I3) slits 10 and $5 \mathrm{~nm}$ RF-5301PC (Shimadzu, Duisburg, Germany). Experiments were also performed without pyrene as reference. Scattered intensity measurements were also performed in DLS Malvern Autosizer 4800 (Malvern, UK) at a scattered angle of $90^{\circ}$ and at a temperature of $22^{\circ} \mathrm{C}$.

\section{Fluorescence Resonance Energy Transfer}

Increasing amounts of siRNA-TAMRA $(0-2.5 \mathrm{nmol} / \mathrm{mL})$ were added to a blank micelle suspension (as described in protocol (iii) but with PLL-FITC). In parallel, the same experiment was performed with PLL-FITC alone. After 15-min incubation, the samples were carried out in a $1 \times 1 \mathrm{~cm}$ path length quartz cuvette (Hellma, Mulheim, Germany) and their spectra were recorded on a RF 5302 Shimadzu spectrofluorometer (Japan) equipped with xenon light source (UXL-150S, Ushio, Tokyo, Japan). Slit used for the micelle experiments were set at 3 and $3 \mathrm{~nm}$ and for the PLL experiments at 3 and $5 \mathrm{~nm}$.

\section{Polarization Experiments}

Solutions of siRNA-TAMRA and suspensions of siRNATAMRA-loaded micelles were submitted to excitation with 
vertically polarized light. The emitted intensities, respectively polarized vertically $\left(I_{\|}\right)$and horizontally $\left(I_{\perp}\right)$ to the reference of the excitation light, were measured successively for each sample. The excitation and emission wavelengths were set to 540 and $583 \mathrm{~nm}$, respectively, with 5 and $10 \mathrm{~nm}$ bandwidths. The steady-state fluorescence polarization factor $p$ was determined according to the following equation:

$$
p=\frac{I_{\|}-G I_{\perp}}{I_{\|}+G I_{\perp}}
$$

where $G$ is the compensating factor for the anisotropic sensitivity of the instrument ( 0.64 in our experimental case).

\section{Bone Marrow-Derived Dendritic Cells Model Mice}

Six to eight-week-old Balb/C mice were obtained from Harlan (France) and C57BL/6 transgenic mice for the expression of eGFP were obtained from The Jackson Laboratory (Bar Harbor, ME, USA). All mice were bred in our own facilities under pathogen-free conditions. All animal experiments were performed according to the French animal protection law as well as after approval and under control of the local authorities.

\section{Bone Marrow-Derived Dendritic Cells Obtention}

The DCs were obtained from BalB/C or C57/BL6 eGFP transgenic mice, according to the Inaba protocol described previously (Charbonnier et al., 2006). Briefly, bone marrow cells were harvested from the femur and tibiae of mice and washed in RPMI 1640 following lyses of red blood cells (Invitrogen, Cergy Pontoise, France). T and B cells were depleted using mouse pan $\mathrm{T}$ and pan B Dynabeads (Dynal Biotech, Cergy Pontoise, France), and monocytes were removed by adhesion in RPMI 1640 with 5\% fetal calf serum (FCS). Remaining cells were cultured in complete medium (RPMI 1640 supplemented with 5\% FCS, $2 \mathrm{mM}$ L-glutamin, $5 \times 10^{-5} \mathrm{M}$ 2-ME, $100 \mathrm{U} / \mathrm{mL}$ penicillin, $100 \mu \mathrm{g} / \mathrm{mL}$ streptomycin, essential amino acids, and $1 \mathrm{mM}$ sodium pyruvate) with $1,000 \mathrm{IU} / \mathrm{mL}$ recombinant murine GM-CSF and 1,000 IU/mL recombinant murine IL-4 (R\&D Systems, Paris, France) at $5 \times 10^{5}$ cells $/ \mathrm{mL}$ in a 24 -well plate. Culture medium was renewed at days 2 and 4 .

\section{siRNA-Loaded Micelles Interaction with DC Confocal Laser Scanning Microscopy}

Confocal laser scanning microscopy was performed on living and fixed cells. To visualize living DCs, FluoroDish ${ }^{\mathrm{TM}}$ (World Precision Instrument, Herts, Germany) were pre-treated with PLL (molecular weight between 30,000 and 70,000 g/mol) for $1 \mathrm{~h}$ and washed twice with RPMI 1640 supplemented with $5 \%$ FCS before adding the cells. Calcein solution (Sigma, Saint Quentin Fallavier, France) at a final concentration of $0.2 \mathrm{mM}$ was added with or without micelles at the same time and then incubated for $4 \mathrm{~h}$ before three washes with PBS and analysis. During analysis, DCs were maintained at $37^{\circ} \mathrm{C}$ under $5 \% \mathrm{CO}_{2}$. To visualize the fixed cells, lab-Teck slides (Nunc, Germany) were precoated with PLL for $1 \mathrm{~h}$ at RT and washed twice with sterile water and twice with RPMI 1640 supplemented with 5\% FCS. Cells were adhered for $30 \mathrm{~min}$ at $37^{\circ} \mathrm{C}$ on the PLL-coated slides and fixed with $4 \%$ paraformaldehyde for $15 \mathrm{~min}$ at RT. After two washing steps, cells were incubated with $0.5 \mathrm{M}$ $\mathrm{NH}_{4} \mathrm{Cl}$ for $15 \mathrm{~min}$ at $\mathrm{RT}$ and washed twice with PBS. Slides were mounted according to the manufacturer's specifications with Vectashield mounting medium containing 4',6-diamidino2-phenylindole (DAPI) (Cliniscience, Maubeuge, France) for nuclei staining.

Cells or slides were examined by confocal laser scanning microscopy using a Zeiss 5 Live Duo (Carl Zeiss Microscope Systems, Gottingen, Germany) and the pictures were analyzed using LSM Image Browser (Carl Zeiss Microscope Systems, Gottingen, Germany).

\section{Fluorescence Microscopy}

After 24-h incubation with siRNA-loaded micelles, cells were observed on Axiovert 200M (Carl Zeiss Microscope Systems, Gottingen, Germany) under $5 \% \mathrm{CO}_{2}$ and at $37^{\circ} \mathrm{C}$. The pictures were then analyzed using MetaMorph soft (Molecular Device, Union City, CA, USA).

\section{RESULTS}

\section{siRNA-Loaded Micelles Characterization}

Among the three protocols tested to prepare the siRNAloaded micelles, all of them allowed to reach an encapsulation yield of $100 \%$ whatever the concentrations of added siRNA $(0.1-5 \mathrm{nmol} / \mathrm{mL})$. This high encapsulation yield, already described in the literature (Katas \& Alpar, 2006), was because of the strong affinity of negatively charged siRNA for the positively charged PLL, and the low amount of siRNA to entrap. The hydrodynamic diameters of the formulated micelles (assessed in PBS $150 \mathrm{mM}, \mathrm{pH}$ 7.4) increased progressively with the addition of higher amounts of siRNA. Indeed, for siRNA-unloaded micelles, the Dh was evaluated at $60 \mathrm{~nm}$ and reached 64,75 , and $86 \mathrm{~nm}$ for siRNA concentration of $0.5,1.5$, and $2.5 \mathrm{nmol} / \mathrm{L}$, respectively. These results suggest the effective entrapment of the nucleotides into the micelles. All the formulations were characterized by a polydispersity index of 0.2 meaning a homogeneous population. Thus, siRNA-loaded micelles characteristics, in agreement with our specifications ( $\mathrm{Dh}<100 \mathrm{~nm}$ and low polydispersity index), appear favorable to an effective and "stressless" endocytosis by the DCs.

siRNA-loaded micelle formulation was based on a tripartite association between a double-hydrophilic block copolymer, PMAA- $b$-POE, a counter polyion, PLL and the siRNA. In order to understand this organization, and especially the interactions 


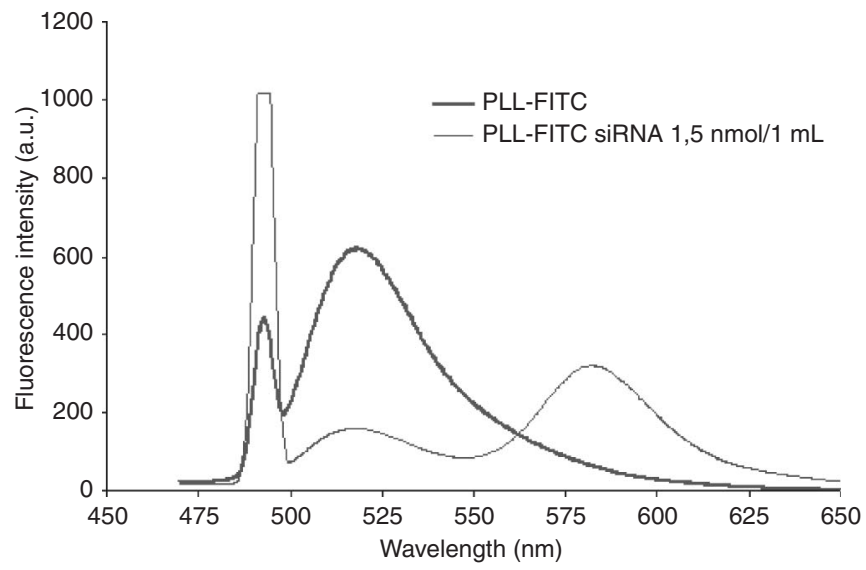

FIGURE 1. FRET spectra between PLL-FITC and siRNA-TAMRA.

between PLL (positively charged) and the siRNA (negatively charged), a fluorescence resonance energy transfer (FRET) study was carried out. FRET is defined as a nonradiative energy transfer because of a dipole-dipole interaction between the donor and the acceptor, according to Förster (1959). FRET efficiency is a process depending on the spectral properties and the inter-fluorophore distance of the pair (usually between 1 and $10 \mathrm{~nm}$ ). Experiments were performed using FITC-linked PLL as the donor and TAMRA-labelled siRNA as the acceptor. In the first part, the spectra of the unformulated compounds were drawn (Figure 1). For FITC-linked PLL, the excitation and emission peaks were recorded at 490 and $517 \mathrm{~nm}$, respec- tively. After addition of TAMRA-labelled siRNA $(1.5 \mathrm{nmol} / \mathrm{mL})$ to FITC-linked PLL solution, a new peak appeared at $583 \mathrm{~nm}$ because of TAMRA. Moreover, the intensity of the emission peak of FITC $(517 \mathrm{~nm})$ was reduced in the presence of TAMRA (620 vs. 159 arbitrary unit: a.u.), demonstrating the energy transfer between the two fluorophores. In the second part of the study, the same experiments were performed with blank micelles in the presence of increasing amounts of siRNA (from 0 to $2.5 \mathrm{nmol} / \mathrm{mL}$ ) (Figure 2). Again, without siRNA, only one emission peak was observed $(517 \mathrm{~nm})$ with an intensity of 808 a.u. After the micelle incubation with siRNATAMRA, the FITC emission peak intensity decreased and TAMRA emission peak appeared $(583 \mathrm{~nm})$. With the increasing concentrations of siRNA, the emission peak intensity of FITC progressively decreased from 808 to 423 a.u. In parallel, the intensity of the TAMRA emission peak increased up to 176 a.u. for a $2.5 \mathrm{nmol} / \mathrm{mL}$ siRNA-TAMRA final concentration. These experiments underscored the interactions between PLL-FITC and siRNA-TAMRA and showed the siRNA tropism for the micelle core (Boudier et al., 2009).

The fluorescence anisotropy of siRNA-TAMRA was studied in order to monitor its motion in function of its microenvironment. In this way, $p$, the fluorescence polarization factor of free siRNA-TAMRA or micelle-loaded siRNA-TAMRA was measured. With free siRNA-TAMRA, $p$ was measured at 0.22 whereas in the tripartite association (siRNA-TAMRA-loaded micelles), $p$-value increased up to 0.27 . This increase of $p$ proved that siRNA motion was reduced and consequently strongly interacted with the micelles. Both results, FRET and fluorescence

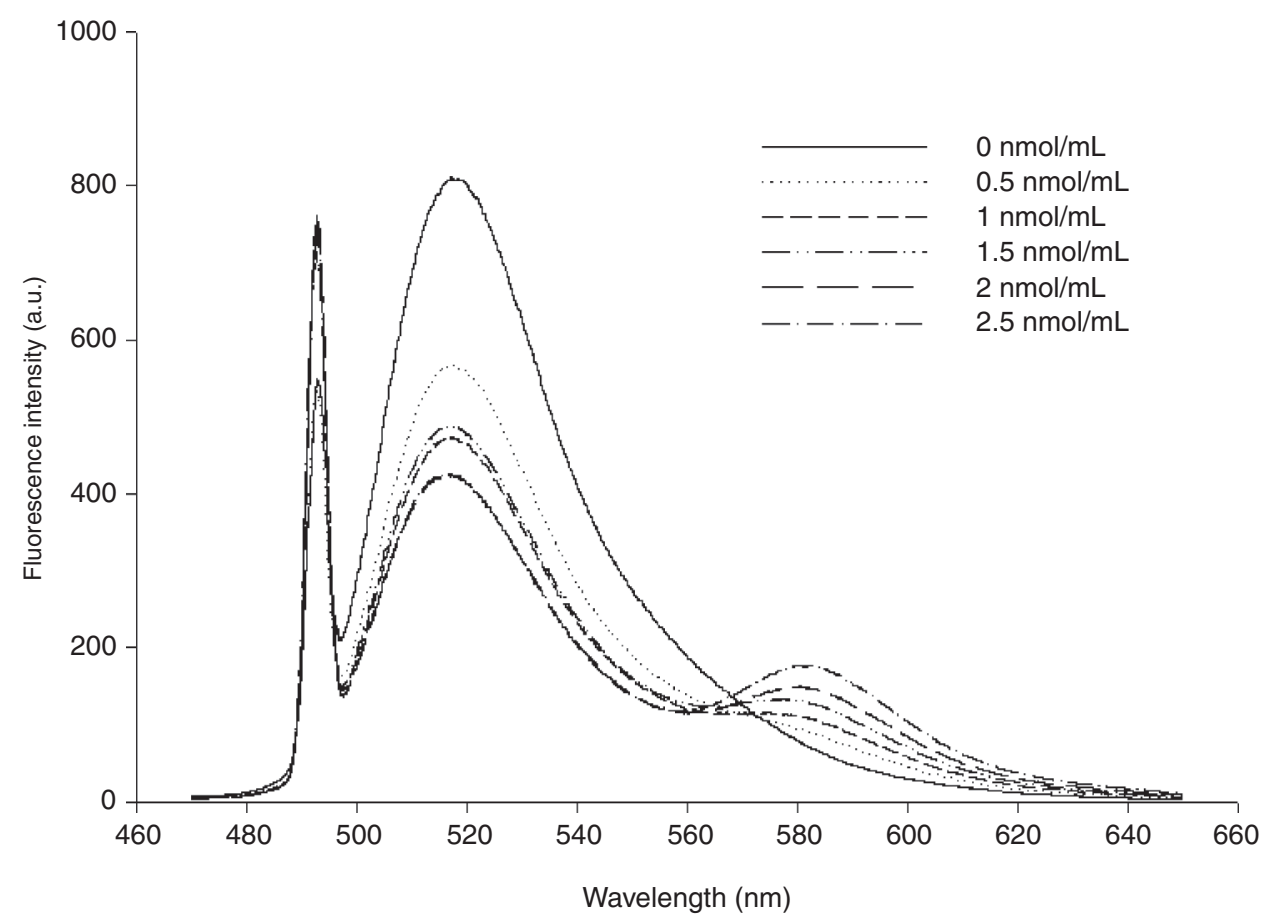

FIGURE 2. FRET spectra between blank micelles formulated with PLL-FITC and siRNA-TAMRA at various concentrations. 
polarization, confirmed the hypothesis that TAMRA-labelled siRNA should be located into the core of the micelle.

\section{siRNA-Loaded Micelles Properties}

Two main properties are required by micelles: $\mathrm{pH}$ sensitivity and the ability to endosomal escape. These properties have to allow siRNA to follow an appropriate intracellular trafficking in order to induce mRNA interference. Indeed after their endocytosis, the micelles disassembly (in acidic conditions) to free siRNA which has to escape from the endosome in order to reach the cytoplasm of the transfected cell. Then it could interact with the RISC multi-protein complex and induce the silencing of the targeted gene (Zhang et al., 2007).

The $\mathrm{pH}$ sensitivity of the siRNA-loaded micelles was obtained by the appropriate choice of the copolymer and its counterion (Boudier et al., 2009) and assessed in vitro by measuring the evolution of the micelle size using DLS at two $\mathrm{pH}$ values (buffered solutions at $\mathrm{pH} 5$ and 7.4). The scattered intensity at physiological $\mathrm{pH}$ was measured at $463 \mathrm{kcts} / \mathrm{s}$. This value drastically decreased to $45 \mathrm{kcts} / \mathrm{s}$ in acidic conditions ( $\mathrm{pH}$ 5). This intensity decrease demonstrated the complete dissociation of the micelle under acidic conditions validating their $\mathrm{pH}$ sensitivity.

The ability of the micelles to escape from the endosomes was imputed to the presence of the polymethacrylic acids (PMAA- $b$-POE) in their formulation. Indeed, these polymers are known to destabilize the membrane bilayers as a consequence of their pH-triggered conformational change (Yessine \& Leroux, 2004). Thus, the conformation of our PMAA- $b$-POE copolymer in function of the $\mathrm{pH}$ was studied using DLS and fluorescence with pyrene incorporation (Figure 3). In DLS, from $\mathrm{pH}$ 7.8 to 4.2 , the scattered intensities were low (less than 20 a.u.) suggesting a perfectly soluble copolymer. Then, from $\mathrm{pH} 4.2$ to 3, they rapidly increased from 20 to 170 a.u., meaning the organization of polymer under globular particles. The fluorescent spectra of the pyrene and particularly the intensity of its first and third vibronic bands (I1 and I3, respectively) are a direct function of the microenvironment polarity: the I1/I3 ratio evolves in the same direction (Kalyanasundaram \& Thomas, 1977; Lochmüller \& Wenzel, 1990). We could observe that the I1/I3 ratio decreased from $\mathrm{pH} 7.8$ (I1/I3 = $1.58)$ to $4.6(\mathrm{I} 1 / \mathrm{I} 3=1.38)$ meaning the appearance of hydrophobic domains. Then under acidic $\mathrm{pH}$ from 4.8 to 2.5 , the value was stabilized at 1.36 . These results are in accordance with previous studies with PMAA- $b$-POE copolymers, also known to associate themselves under acidic conditions by intra- and intermolecular hydrogen bonds between carboxylic groups of PMAA and the POE units (Holappa, Andersson, Kantonen, Plattner, \& Tenhu, 2003). Thus, these results confirm that PMAA- $b$-POE copolymers underwent conformational changes from soluble objects at physiological $\mathrm{pH}$ to more hydrophobic globular ones in acidic environment according to what was previously described (Yessine, Lafleur, Meier, Petereit, \& Leroux, 2003). Taking into account the fact that the $\mathrm{pH}$ in the DC endosomes following lysosome fusion is acidic and is about 5 (Hart, 1997), we could suppose that for this value, PMAA- $b$-POE has already adopted a globular conformation and could be able to destabilize membranes of the late endosomes.

\section{siRNA-Loaded Micelles Interaction with Dendritic Cells}

In order to investigate the intracellular trafficking of siRNAloaded micelles, different experiments were performed with DCs. First, the endocytosis step was studied by incubating

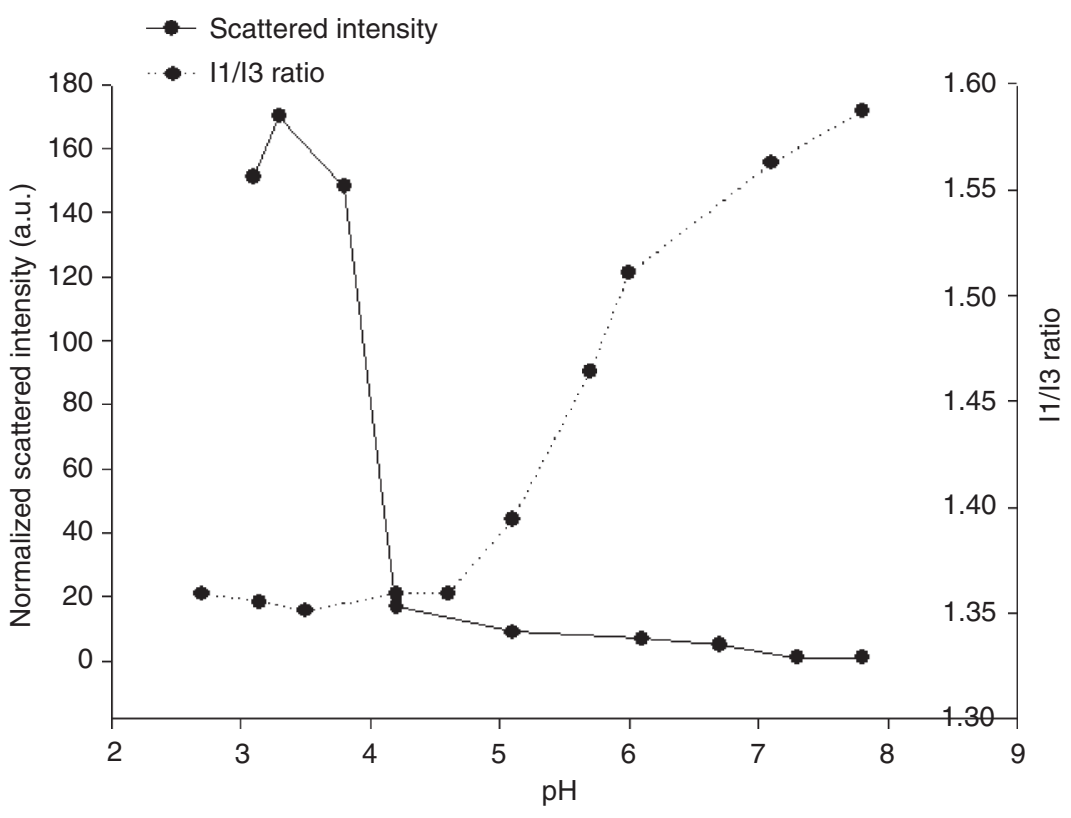

FIGURE 3. Copolymer behavior as a function of the $\mathrm{pH}$ evaluated by the scattered intensity (solid line) and the I1/I3 ratio related to pyrene incorporation (dotted line). 


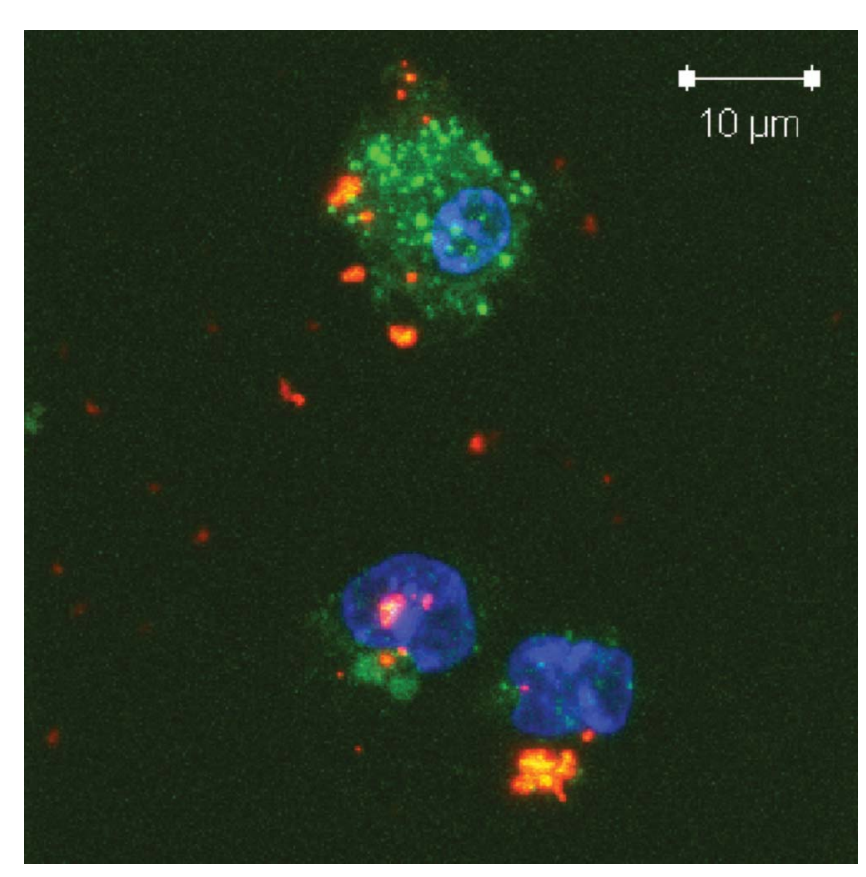

FIGURE 4. Confocal microscopy of dendritic cells incubated $1 \mathrm{~h}$ with calcein (green) and siRNA-TAMRA-loaded micelles (red). Nuclei were labelled with DAPI (blue). Scale bar $=10 \mu \mathrm{m}$

siRNA-TAMRA-loaded micelles with the DCs in the presence of calcein, known as a membrane-impermeant fluorescent probe (Hu et al., 2007) (Figure 4). After 1-h incubation, the endocytosis of the probe within the endosomal vesicles was suggested by the punctuated green fluorescent signal observed by confocal microscopy. The TAMRA-labelled-siRNA-loaded micelles were shown to co-localize within the endolysosomal compartments with the presence of yellow dots. Thus, siRNAloaded micelles were successfully endocytosed as it was already shown for blank micelles (Boudier et al., 2009)

To confirm the micelle property of endosomal disruption (thanks to the PMAA- $b$-POE), experiments were done on living DCs with or without blank micelles (Figure 5) using calcein as probe. After 4-h incubation, a homogeneous signal of calcein was observed into all the cytoplasm due to the escape from the endosomes to the cytosol (Figure 5A). In contrast, without micelle, a punctuated fluorescent signal of calcein was observed. Thus, blank micelles were able to destabilize endosomal membranes. To check this phenomenon with siRNAloaded micelles, DCs were incubated with micelles formulated with PLL-FITC and siRNA-TAMRA and visualized by confocal microscopy at different times (Figure 6A and B, respectively). In the first hours, some vesicles containing siRNA and PLL were clearly individualized into the cell whereas after 24-h incubation, the two fluorescent signals were visualized into the cytoplasm without any preferred site. Altogether these results suggested a disassembly of the micelles into the endosomes, followed by a destabilization of their membrane via the PMAA$b$-POE allowing the release of siRNA into the cytoplasm.

\section{eGFP Silencing Using siRNA-Loaded Micelles}

To validate the use of such micelles as siRNA vectors to engineer DCs, the micelles were prepared with siRNA targeting eGFP transcript as proof of concept and were added on DCs derived from eGFP transgenic mice. The cells were visualized by fluorescence microscopy $48 \mathrm{~h}$ after their incubation either with eGFP targeting siRNA-loaded micelles or with irrelevant siRNA-loaded micelles (control) (concentrations of $2.5 \mathrm{nM}$ of siRNA and $3.25 \mu \mathrm{g} / \mathrm{mL}$ of copolymers in each case) (Figure 7). In these experiments, the cells treated with eGFP targeting siRNA-loaded micelles showed a decreased expression of eGFP fluorescent signal compared to control cells incubated with irrelevant siRNA-loaded micelles. The present result suggested a silencing of the eGFP expression in the engineered DCs with siRNA-loaded particles validating the use of such micelles as a vehicle to deliver functional siRNA in primary DC.

\section{DISCUSSION}

Although DCs have a pivotal role in the immune response and are of great interest for therapy (Banchereau \& Palucka, 2005), up to now, only few studies have been published using
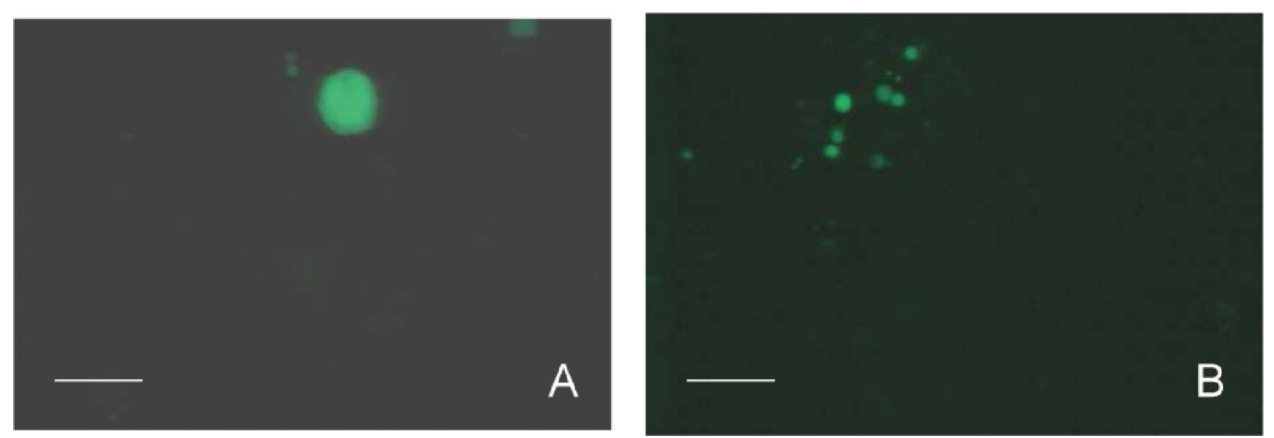

FIGURE 5. Confocal microscopy on living dendritic cells incubated with calcein and with $(\mathrm{A})$ or without $(\mathrm{B})$ siRNA-TAMRA-loaded micelles, scale bar $=20$ $\mu \mathrm{m}$. Scale bar $=10 \mu \mathrm{m}$. 

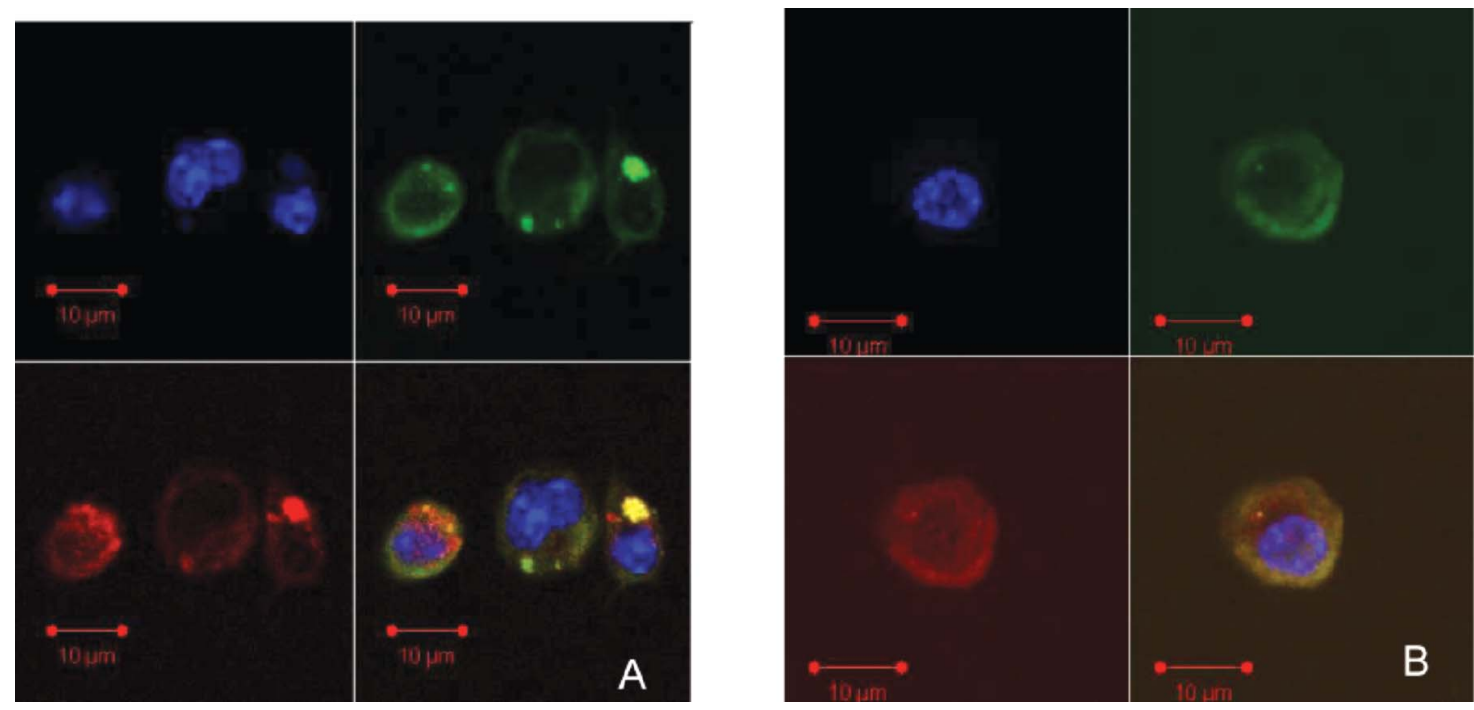

FIGURE 6. Confocal microscopy of dendritic cells after a short time (A) or 24-h (B) incubation with siRNA-TAMRA-loaded micelles formulated with PLL-FITC.
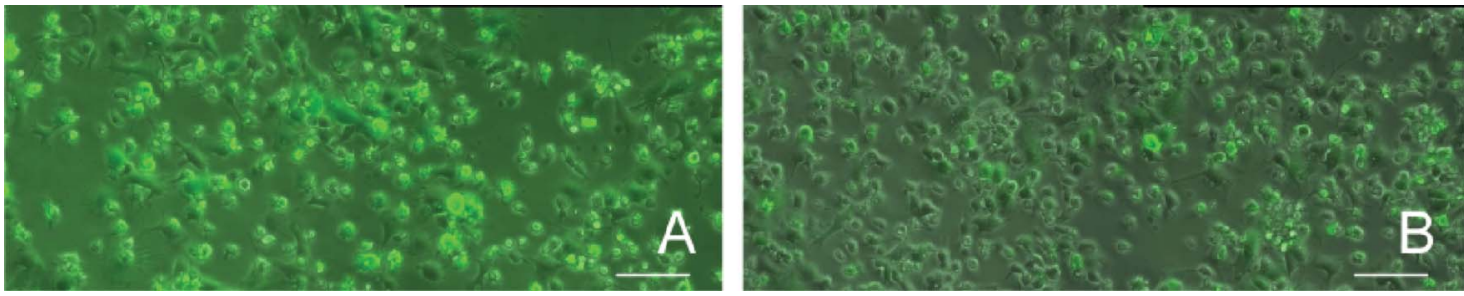

FIGURE 7. Fluorescence microscopy of $\mathrm{GFFP}^{+}$dendritic cells after 48-h incubation with control siRNA-loaded micelles (A) or with eGFP targeting siRNAloaded micelles (B). Scale bar $=40 \mu \mathrm{m}$.

nonviral vectors encapsulating siRNA to silence target gene in these particular cells (Hill et al., 2003; Kim et al., 2005; Suzuki et al., 2008). This observation is notable since interference has been studied in many cell lines or whole organism either to assess the role of the targeted gene or for a therapeutic potential (Zhang et al., 2007). Thus, even if the application of siRNA in DCs seems to be a challenge, it is of clinical relevance since the plasticity of such immune cell is crucial in the immune response (Van Duivenvoorde, Van Mierlo, Boonman, \& Toes, 2006). The aim of our work was to formulate a siRNA vector for DCs and to evaluate its intracellular trafficking especially in terms of endosomal escape ability and release of siRNA.

Polyion complex micelles have already been used to formulate siRNA or oligonucleotides because of their core made of a coacervat (Van Der Burgh, De Keizer, \& Cohen Stuart, 2004). Indeed, pegylated siRNA and PLL led to micelles with encouraging results (Nishiyama \& Kataoka, 2006). The originality of our study lies on a tripartite association between the siRNA, the counter polyion, and the copolymer. Only one work with such an association was described in the literature, but with oligonucleotide and two types of diblock copolymers (Yessine, Dufresne, Meier, Petereit, \& Leroux, 2007). The choice of a tripartite association aimed at modulating the siRNA as well as the micelle concentrations because the siRNA was not used as a counter polyion.

The physicochemistry study of the micelle using fluorescent probes revealed privileged interactions between siRNA and PLL and furthermore a siRNA localization into the micelle core. Both results allow us to understand the high encapsulation yield. The $\mathrm{pH}$ sensitivity of the system was guaranteed by the polymethacrylic acid part of the copolymer which makes possible the siRNA delivery in a controlled way into the late acidic endosomes because of the disassembly of the micelles. The siRNA entrapment into micelles did not alter this stimulus dependence. siRNA-loaded micelles showed the same biocompatible properties than blank micelles, making possible the work on DCs without altering their maturation state (Boudier et al., 2009) and thus widening their application field.

On the other hand, the destabilization of the endosomal membranes and as a consequence the siRNA release into the cytoplasm, which is a key event to allow the silencing of the protein expression was assessed. This parameter was attributed to the copolymer-specific organization under acidic conditions. Indeed, the conformation of the PMAA- $b$-POE varied with the 
pH: under acidic conditions, the polymethacrylic acid chains underwent hydrophobic interactions as well as hydrogen bonding implying the formation of insoluble objects. Such particles might have been able to interact with endosomal membrane, probably via phosphatidylcholine molecules particularly concentrated in such organelles as suggested in the literature (Yessine \& Leroux, 2004).

Finally, we validated the use of the $\mathrm{pH}$-sensitive copolymer micelle as a vector for siRNA to silence a targeted gene in DCs. Indeed, our results using siRNA targeting the expression of eGFP as proof of concept underscore the potential of such micelles as a cytocompatible vehicle to deliver functional siRNA into DC as a therapeutic molecule. Future studies will focus on the silencing of co-stimulatory molecules or proinflammatory cytokines in order to be able to dampen the immunogenicity of the DC triggering the education of regulatory $\mathrm{T}$ lymphocytes. In contrast, another area of research will aim to target inhibitory molecule in order to amplify the activation of antigen-specific $\mathrm{T}$ lymphocyte in order to enhance immune response against cancer cells. Quantitative analyses will be needed to assess the silencing of the targeted genes and a lot of effort will be made to optimize the siRNA concentration to obtain the best therapeutic effect.

This study underscore the potential of tripartite $\mathrm{pH}$-sensitive polyion complex micelles as original tools to formulate therapeutic siRNA for DC engineering in order to maintain the homeostasis of the immune response.

\section{CONCLUSION}

In this study, a tripartite association was studied to induce RNA interference in DCs. This new vector made of a doublehydrophilic block copolymer, PLL, and siRNA was formulated and tested on primary murine DCs. siRNA was successfully entrapped into the polyion complex micelles. Following endocytosis of the siRNA-loaded micelles, endosomal disruption allowed the release of the siRNA into the cytosol, and efficient silencing of the targeted gene. These results are of great therapeutic interest since only few studies deal with the silencing of target genes in DCs.

\section{ACKNOWLEDGMENTS}

The authors are willing to thank Montpellier RIO imaging platform to have put confocal laser scanning microscopy and fluorescence microscopy at our disposal and P. Peralta for technical support.

\section{REFERENCES}

Akhtar, S., \& Benter, I. F. (2007). Nonviral delivery of synthetic siRNAs in vivo. J. Clin. Invest., 117, 3623-3632.

Banchereau, J., \& Palucka, K. A. (2005). Dendritic cells as therapeutic vaccines against cancer. Nature, 5, 296-306.

Boudier, A., Aubert-Pouëssel, A., Louis-Plence, P., Gérardin, C., Jorgensen, C., Devoisselle, J.-M., \& Bégu, S. (2009). The control of the dendritic cell maturation by $\mathrm{pH}$-sensitive polyion complex micelles. Biomaterials, 30 , 233-241.

Charbonnier, L.-M., Van Duivenvoorde, L. M., Apparailly, F., Cantos, C., Han, W. G. H., Noël, D., Dupperay, C., Huizinga, T. W. J., Toes, R. E. M., Jorgensen, C., \& Louis-Plence, P. (2006). Immature dendritic cells suppress collagen-induced arthritis by in vivo expansion of $\mathrm{CD} 49 \mathrm{~b}+$ regulatory $\mathrm{T}$ cells. J. Immunol., 177, 3806-3813.

Fire, A., Xu, S., Montgomery, M. K., Kostas, S. A., Driver, S. E., \& Mello, C. C. (1998). Potent and specific genetic interference by double-stranded RNA in Caenorrhabditis elegans. Nature, 391, 806-810.

Förster, T. (1959). Transfer mechanisms of electronic excitation. Discuss. Faraday Soc., 27, 7-17.

Hart, D. (1997). Dendritic cells: Unique leucocyte populations which control the primary immune response. Blood, 90, 3245-3287.

Hill, J. A., Ichim, T. E., Kusznieruk, K. P., Li, M., Huang, X., Yan, X., Zhong, R., Cairns, E., Bell, D. A., \& Wei-Ping, M. (2003). Immune modulation by silencing Il-12 production in dendritic cells using small interfering RNA. $J$. Immunol., 171, 691-696.

Holappa, S., Andersson, T., Kantonen, L., Plattner, P., \& Tenhu, H. (2003). Soluble polyelectrolyte complexes composed of poly(ethylene oxide)-blockpoly(sodium methacrylate) and poly(methacryloyloxyethyl trimethylammonium chloride). Polymer, 44, 7907-7916.

Hu, Y., Litwin, T., Nagaraja, A. R., Kwong, B., Katz, J., Watson, N., \& Irvine, D. J. (2007). Cytosolic delivery of membrane-impermeable molecules in dendritic cells using $\mathrm{pH}$-responsive core-shell nanoparticles. Nano Lett., 7 , 3056-3064

Kakizawa, Y., \& Kataoka, K. (2002). Block copolymer micelles for delivery of gene and related compounds. Adv. Drug Deliv. Rev., 54, 203-222.

Kalyanasundaram, K., \& Thomas, J. K. (1977). Environmental effects on vibronic band intensities in pyrene monomer fluorescence and their application in studies of micellar systems. J. Am. Chem. Soc., 99, 2039-2044.

Katas, H., \& Alpar, H. O. (2006). Development and characterisation of chitosan nanoparticles for siRNA delivery. J. Control. Release, 115, 216-225.

Kim, W. T., Lee, J. H., He, L., Boyd, D. A. K., Hardwick, J. M., Hung, C.-F., $\& \mathrm{Wu}$, T.-C. (2005). Modification of professional antigen-presenting cells with small interfering RNA in vivo to enhance cancer vaccine potency. Cancer Res., 65, 309-316.

Lavasanifar, A., Samuel, J., \& Kwon, G. S. (2002). Poly(ethylene oxide)block-poly(-amino acid) micelles for drug delivery. Adv. Drug Deliv. Rev., 54, 169-190.

Li, C., Parker, X. A., Menocal, E., Xiang, S., Borodyansky, L., \& Fruehauf, J. H. (2006). Delivery of RNA interference. Cell cycle, 5, 2103-2109.

Li, M., Zhang, X., Zheng, X., Lian, D., Zhang, Z. X., Ge, W., Yang, J., Vladau C., Suzuki, M., Chen, D., Zhong, R., Garcia, B., Jevnikar, A. M., Min, W. P. (2007). Immune modulation and tolerance induction by RelB-silenced dendritic cells through RNA interference. J. Immunol., 178, 5480-5487.

Lochmüller, C. H., \& Wenzel, T. J. J. (1990). Spectroscopic studies of pyrene at silica interfaces. J. Phys. Chem., 94, 4230-4235.

Napoli, C., Lemieux, C., \& Jorgensen, R. (1990). Introduction of a chimeric chalcone synthase gene into petunia results in reversible co-suppression of homologous genes in trans. Plant Cell, 2, 279-289.

Nishiyama, N., \& Kataoka, K. (2006). Current state, achievements, and future prospects of polymeric micelles as nanocarriers for drug and gene delivery. Pharmacol. Therapeut., 112, 630-648.

Oishi, M., Hayama, T., Akiyama, Y., Takae, S., Harada, A., Yamasaki, Y., Nagatsugi, F., Sasaki S., Nagasaki, Y., \& Kataoka, K. (2005). Supramolecular assemblies for the cytoplasmic delivery of antisense oligonucleotide: Polyion complex (PIC) micelles based on poly(ethyleneglycol)-SS-oligonucleotide conjugate. Biomacromolecules, 6, 2449-2454.

Steinman, R., \& Banchereau, J. (2007). Taking dendritic cells into medicine. Nature, 449, 419-426.

Suzuki, M., Zheng, X., Zhang, X., Li, M., Vladau, C., Ichim, T. E., Sun, H., Min, L. R., Garcia, B., \& Min, W. P. (2008). Novel vaccination for allergy through gene silencing of CD40 using small interfering RNA. J. Immunol., 180, 8461-8469.

Van Der Burgh, S., De Keizer, A., \& Cohen Stuart, M. A. (2004). Complex coacervation core micelles. Colloidal stability and aggregation mechanism. Langmuir, 20, 1073-1084. 
Van Duivenvoorde, L. M., Van Mierlo, G. J. D., Boonman, Z. F. H. M., \& Toes, R. E. M. (2006). Dendritic cells: Vehicles for tolerance induction and prevention of autoimmune diseases. Immunobiology, 211, 627-632.

Yessine, M.-A., Dufresne, M.-H., Meier, C., Petereit, H.-U., \& Leroux, J.-C. (2007). Proton-actuated membrane-destabilizing polyion complex micelles. Bioconjug. Chem., 18, 1010-1014.

Yessine, M.-A., Lafleur, M., Meier, C., Petereit, H.-U., \& Leroux, J.-C. (2003). Characterization of the membrane-destabilizing properties of different
pH-sensitive methacrylic acid copolymers. Biochim. Biophys. Acta, 1613, 28-38.

Yessine, M.-A., \& Leroux, J.-C. (2004). Membrane-destabilizing polyanions: Interaction with lipid bilayers and endosomal escape of biomacromolecules. Adv. Drug Deliv. Rev., 56, 999-1021.

Zhang, S., Zhao, B., Jiang, H., Wang, B., \& Ma, B. (2007). Cationic lipids and polymers mediated vectors for delivery of siRNA. J. Control. Release, 123, 1-10. 